\title{
Phase formation and ferrimagnetism of $\mathrm{GdCogSi}_{4}$
}

\author{
M El-Hagary ${ }^{1,2}$, H Michor ${ }^{1,8}, \mathbf{S}$ Özcan ${ }^{1}$, M Giovannini $^{3}$, A Matar $^{4}$, \\ Z Heiba ${ }^{5}$, P Kerschl ${ }^{1}$, M Schönhart ${ }^{1}$, E Bauer ${ }^{1}$, R Grössinger ${ }^{1}$, \\ G Hilscher $^{1}$, J Freudenberger ${ }^{6}$ and $\mathbf{H}$ Rosner $^{7}$ \\ ${ }^{1}$ Institut für Festkörperphysik, Technische Universität Wien, Wiedner Hauptstrasse 8-10, A-1040 \\ Wien, Austria \\ 2 College of Science, Physics Department, Qassim University, Buraidah 81999, Kingdom of Saudi \\ Arabia \\ ${ }^{3}$ Dipartimento di Chimica e Chimica Industriale, Università di Genova, Via Dodecaneso 31, \\ I-16146 Genova, Italy \\ ${ }^{4}$ Physics Department, Faculty of Science, Helwan University, Helwan, Cairo, Egypt \\ ${ }^{5}$ Physics Department, Faculty of Science, Ain Shams University, Cairo, Egypt \\ ${ }^{6}$ Leibniz-Institut für Festköper- und Werkstoffforschung, IFW Dresden, D-01171 Dresden, \\ Germany \\ ${ }^{7}$ Max-Planck Institut für Chemische Physik fester Stoffe, Nöthnitzer Strasse 40, D-01187 \\ Dresden, Germany \\ E-mail: michor@ifp.tuwien.ac.at
}

Received 12 January 2006

Published 26 April 2006

Online at stacks.iop.org/JPhysCM/18/4567

\begin{abstract}
The phase relations of the $\mathrm{GdCo}_{13-x} \mathrm{Si}_{x}$ system have been studied by means of scanning microscopy, microprobe analysis and $\mathrm{x}$-ray diffraction. Single-phase samples $\mathrm{GdCO}_{9 \pm \delta} \mathrm{Si}_{4 \mp \delta}$ (structure type $\mathrm{LaFe}_{9} \mathrm{Si}_{4}$ with space group $I 4 / \mathrm{mcm}$ ) are formed in a narrow composition range $|\delta| \leqslant 0.2$, where $\mathrm{GdCo}_{9} \mathrm{Si}_{4}$ forms as a fully ordered ternary compound. The magnetic properties of $\mathrm{GdCo}_{9} \mathrm{Si}_{4}$ have been investigated by ac susceptibility, magnetization, specific heat and resistivity measurements. These experiments reveal ferrimagnetism below about $47 \mathrm{~K}$, which is analysed in terms of a two-sublattice molecular field model with a local moment Gd subsystem coupling antiparallel to the itinerant ferromagnetic Co $3 \mathrm{~d}$ sublattice. The $3 \mathrm{~d}-3 \mathrm{~d}$ exchange of the latter is the driving force for the onset of long range magnetic order. The validity of the twosublattice model is demonstrated by high field measurements, showing that the ferrimagnetic coupling starts to break up at a lower critical field $\mu_{0} H_{1} \simeq 27 \mathrm{~T}$. The magnetic ground state of $\mathrm{GdCo}_{9} \mathrm{Si}_{4}$ has been analysed on a microscopic basis via LSDA electronic structure calculations.
\end{abstract}

(Some figures in this article are in colour only in the electronic version)

8 Author to whom any correspondence should be addressed. 


\section{Introduction}

Rare earth intermetallic compounds $\mathrm{RT}_{13-x} \mathrm{M}_{x}(\mathrm{R}=$ rare earth, $\mathrm{T}=\mathrm{Co}, \mathrm{Fe}$ and $\mathrm{M}=\mathrm{Si}$, Al) have gained attention as potential permanent magnetic materials, highly efficient magnetocaloric materials and giant isotropic magnetostriction materials [1-4]. Besides these applied aspects there are important features motivating basic research, namely the occurrence of itinerant electron metamagnetism in $\mathrm{LaCo}_{9} \mathrm{Si}_{4}$ and weak itinerant ferromagnetism in $\mathrm{YCo}_{9} \mathrm{Si}_{4}[5,6]$. Within the solid solution $\mathrm{LaCo}_{13-x} \mathrm{Si}_{x}$, ferromagnetism vanishes near the stoichiometric composition $\mathrm{LaCo}_{9} \mathrm{Si}_{4}$, where full translational symmetry (space group $I 4 / \mathrm{mcm}$ ) is confirmed by single-crystal x-ray diffractometry [5, 7]. $\mathrm{LaCo}_{9} \mathrm{Si}_{4}$ is a strongly exchange-enhanced Pauli paramagnet with a rather large Sommerfeld coefficient of the electronic specific heat $\gamma \simeq 200 \mathrm{~mJ} \mathrm{~mol}^{-1} \mathrm{~K}^{-2}$ associated with a spin fluctuation mass enhancement $\lambda_{\text {sf }} \sim 3.3$. The close vicinity of a ferromagnetic instability is seen in external magnetic fields as low as $3.5 \mathrm{~T}$ for $H \| c$ and $6 \mathrm{~T}$ for $H \perp c$ where $\mathrm{LaCo}_{9} \mathrm{Si}_{4}$ undergoes an itinerant electron metamagnetic phase transition where essentially one site (16k) out of three inequivalent crystallographic sites occupied by Co becomes magnetic while the induced moments remain rather small on the other two sites (161 and 4d) [5].

For the related isostructural compound $\mathrm{YCo}_{9} \mathrm{Si}_{4}$, electronic structure calculations indicate very similar band features related to the Co $3 \mathrm{~d}$ bands as in the case of $\mathrm{LaCo}_{9} \mathrm{Si}_{4}$, and in fact specific heat measurements reveal almost exactly the same $\gamma$-value $\simeq 200 \mathrm{~mJ} \mathrm{~mol}^{-1} \mathrm{~K}^{-2}$. Nevertheless, despite the smaller unit cell volume, $\mathrm{YCo}_{9} \mathrm{Si}_{4}$ exhibits spontaneous weak itinerant ferromagnetism below $T_{\mathrm{C}} \simeq 25 \mathrm{~K}[6]$. Other isostructural compounds $\mathrm{RCo}_{9} \mathrm{Si}_{4}$ (except $\mathrm{R}=\mathrm{Ce}$, see [8]) have also been reported to be ferromagnetic, with $T_{\mathrm{C}} \simeq 12-50 \mathrm{~K}$ (see e.g. [9-11]).

In order to explore the interaction between local $4 \mathrm{f}$ moments and the itinerant $3 \mathrm{~d}$ subsystem, we studied the magnetic properties of $\mathrm{GdCo}_{9} \mathrm{Si}_{4}$, which is interesting because of the ${ }^{8} \mathrm{~S}_{7 / 2}$ state of the $4 \mathrm{f}^{7}$ shell with large $S=7 / 2$ spin moment and zero orbital contribution, where in a first approximation crystalline electric field effects are negligible. The re-investigation of the homogeneity region of $\mathrm{GdCo}_{13-x} \mathrm{Si}_{x}$ alloys by scanning electron microscopy (SEM) and electron microprobe analysis (EMPA) is motivated by controversial statements on $\mathrm{GdCo}_{9} \mathrm{Si}_{4}$ by Huang et al [9] reporting the magnetic ordering temperature $T_{\mathrm{C}}$ to be about $50 \mathrm{~K}$, and by Skolozdra et al [12] reporting $T_{\mathrm{C}}=923 \mathrm{~K}$.

\section{Experimental details}

Polycrystalline samples of $\mathrm{GdCo}_{13-x} \mathrm{Si}_{x}$ with nominal compositions from $x=2.0$ to 4.5 were prepared by high frequency induction melting. The starting materials, gadolinium ingots $(99.9 \%)$, cobalt ingots $(99.95 \%$ for the majority of samples and $99.999 \%$ for selected compositions $x=4.0,4.06,4.2)$ and silicon chips $(99.9999 \%)$, were melted together in a two-step procedure: (i) cobalt and silicon were melted together for four times; (ii) the required amounts of Gd were melted together with the precursor CoSi alloys. To ensure homogeneity, the buttons were broken, flipped over and remelted another four times and finally sealed in an evacuated quartz tube and annealed at $1050{ }^{\circ} \mathrm{C}$ for one week. For selected compositions $x=4.0,4.06$, and 4.2, samples have been prepared with $0.02 \mathrm{f}$.u. gadolinium excess in order to inhibit their contamination with traces of the ferromagnetic impurity phase $\mathrm{Co}_{0.92} \mathrm{Si}_{\sim 0.08}$ (see details in section 3.1).

In order to examine the phase formation of $\mathrm{GdCo}_{13-x} \mathrm{Si}_{x}$ and the corresponding homogeneity regions we investigated selected samples by scanning electron microscopy (SEM) and by electron microprobe analysis (EMPA) based on energy dispersive x-ray spectroscopy. 
$\mathrm{Si}$

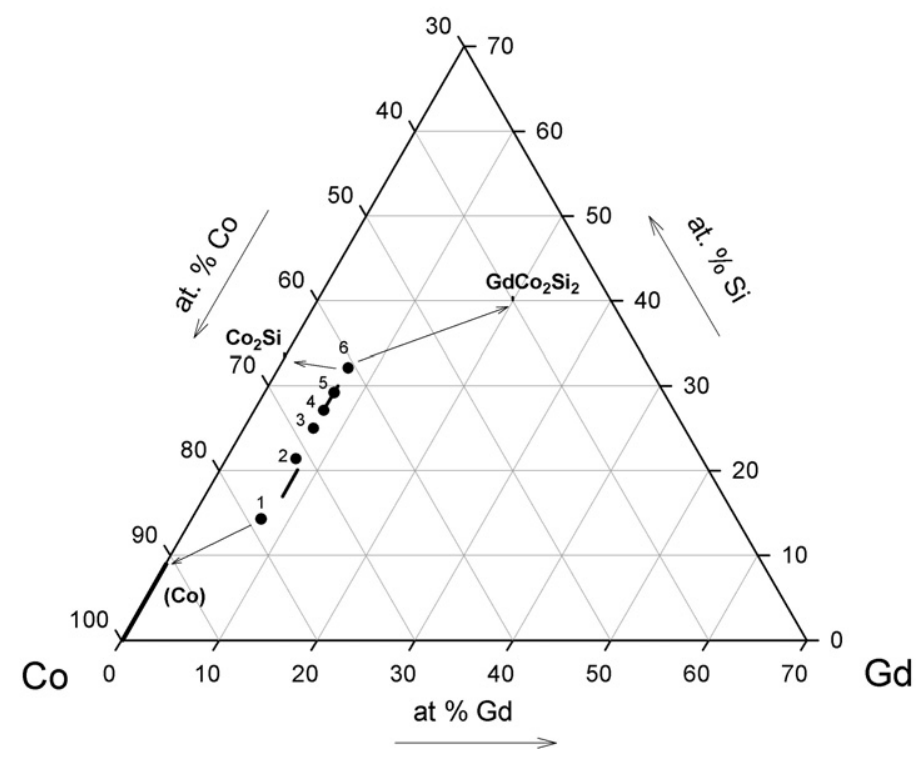

Figure 1. A schematic compositional phase diagram; the two branches of the solid solution are indicated in the phase diagram by full black lines.

Samples for metallographic examination were polished using $\mathrm{SiC}$ and diamond pastes. The pure elements served as standards. Crystallographic characterization was obtained by means of $\mathrm{X}$-ray powder diffraction (XRD) with graphite monochromated $\mathrm{Cu} \mathrm{K} \alpha$ radiation (see [14] for further details).

Magnetic properties were studied by using a commercial calibrated ac susceptometer (Lakeshore 7000) in the temperature range 4.2-300 $\mathrm{K}$ with an ac-field amplitude of $200 \mathrm{~A} \mathrm{~m}^{-1}$ and a frequency of $80 \mathrm{~Hz}$ (low enough to keep eddy current signals negligible in this study). Temperature dependent, $M(T)$, and isothermal magnetization, $M(H)$, measurements were performed in a $6 \mathrm{~T}$ SQUID magnetometer. High field magnetization measurements were performed at the pulsed field facility of the IFW Dresden where fields up to $60 \mathrm{~T}$ are available (see [13] for details of the set-up). Electrical resistivity measurements were performed (4.2$300 \mathrm{~K}$ ) in a He-bath cryostat on bar-shaped samples applying a standard four-probe method with gold pins as voltage and current contacts. Specific heat results were obtained from 2 to $70 \mathrm{~K}$ with a quasi-adiabatic step heating technique with external magnetic fields up to $9 \mathrm{~T}$.

\section{Results and discussion}

\subsection{Phase formation and crystal structure}

EMPA/SEM analysis reveals the presence in the ternary phase diagram of two branches of $\mathrm{Co} / \mathrm{Si}$ substitution solid solutions slightly shifted in Gd composition (see figure 1). The first branch with 8 at.\% Gd forms in the composition range between (75 at.\% Co, 17 at.\% Si) and $\left(72\right.$ at. $\% \mathrm{Co}, 20$ at.\% $\mathrm{Si}$ ) and can be described by the formula $\mathrm{GdCo}_{9-\gamma} \mathrm{Si}_{2+\gamma}$ (in the following referred to as 1:11 type phase). The second branch with 7 at.\% $\mathrm{Gd}$, richer in $\mathrm{Si}$, extends in the range from (66 at.\% Co, 27 at.\% Si) to (63 at.\% Co, 30 at.\% Si), yielding the formula $\mathrm{GdCO}_{9-\delta} \mathrm{Si}_{4+\delta}$ (in the following referred to as 1:13 type phase). The limits of the $\mathrm{GdCo}_{9-\gamma} \mathrm{Si}_{2+\gamma}$ branch have been estimated from the composition of the $\mathrm{Gd}(\mathrm{Co}, \mathrm{Si})_{11}$ phase in samples No 1, No 2 and No 3, yielding $0 \leqslant \gamma \leqslant 0.4$ (see table 1). Samples No 2 and No 3 
Table 1. Results obtained from an EMPA investigation of samples with nominal compositions $\mathrm{GdCo}_{13-x} \mathrm{Si}_{x}$; the locations in the phase diagram are (a) at the lower part of the first branch, (b) the top of the first branch, (c) at the lower part of the second branch, (d) on the second branch and (e) the top of the second branch.

\begin{tabular}{|c|c|c|c|c|}
\hline \multirow[b]{2}{*}{$x$} & \multicolumn{2}{|c|}{ Phases present } & \multirow{2}{*}{$\begin{array}{l}\text { Phase comp. } \\
\text { (at.\% Gd-Co-Si) }\end{array}$} & \multirow{2}{*}{$\begin{array}{l}\text { Location in the } \\
\text { phase diagram }\end{array}$} \\
\hline & Main & Minor & & \\
\hline \multirow[t]{2}{*}{2.0 (No 1) } & bet $1: 11$ & & $8-75-17$ & (a) \\
\hline & & (Co) & $0-92-8$ & \\
\hline \multirow[t]{3}{*}{3.0 (No 2) } & bet $1: 11$ & & $8-72-20$ & (b) \\
\hline & & bet $1: 13$ & $7-66-27$ & (c) \\
\hline & & (Co) & 0-89-11 & \\
\hline \multirow[t]{2}{*}{3.5 (No 3) } & bet $1: 13$ & & $7-66-27$ & (c) \\
\hline & & bet $1: 11$ & $8-72-20$ & (b) \\
\hline 3.8 (No 4) & bet $1: 13$ & (Co) & $7-65-28$ & (d) \\
\hline 4.1 (No 5) & bct $1: 13$ & & $7-64-29$ & (d) \\
\hline \multirow[t]{3}{*}{4.5 (No 6) } & bct $1: 13$ & & $7-63-30$ & (e) \\
\hline & & $\mathrm{Co}_{2} \mathrm{Si}$ & $19-41-40$ & \\
\hline & & $\mathrm{GdCo}_{2} \mathrm{Si}_{2}$ & $1-64-35$ & \\
\hline
\end{tabular}

are located in the area between the two branches, i.e. show the coexistence of the two phases $\mathrm{GdCo}_{9-\gamma} \mathrm{Si}_{2+\gamma}$ and $\mathrm{GdCo}_{9-\delta} \mathrm{Si}_{4+\delta}$ together with traces of the alloy $\mathrm{Co}_{0.92} \mathrm{Si}_{0.08}$. Sample No 5 reveals single-phase $\mathrm{GdCo}_{9-\delta} \mathrm{Si}_{4+\delta}$ with an atom composition of 7 at.\% $\mathrm{Gd}, 64$ at.\% $\mathrm{Co}$ and 29 at.\% Si and, finally, No 6 exhibits three phases: $\mathrm{GdCo}_{9-\delta} \mathrm{Si}_{4+\delta}, \mathrm{GdCo}_{2} \mathrm{Si}_{2}$ and $\mathrm{Co}_{2} \mathrm{Si}$. The approximate limits of the $\mathrm{GdCo}_{9-\delta} \mathrm{Si}_{4+\delta}$ branch have been estimated from the composition of the $\mathrm{Gd}(\mathrm{Co}, \mathrm{Si})_{13}$ majority phase in samples No 3 and No 6 , yielding $-0.2 \leqslant \delta \leqslant 0.2$.

A schematic compositional phase diagram shown in figure 1 displays the SEM/EMPA results (summarized in table 1), i.e. the location of the nominal compositions of the investigated samples, the two branches of the solid solution (they are indicated in the phase diagram by full lines) and the phases obtained.

The above results are corroborated by $x$-ray refinements. XRD patterns of the alloys prepared along the composition line $\mathrm{GdCo}_{13-x} \mathrm{Si}_{x}(2 \leqslant x \leqslant 4.5)$ reveal essentially two types of crystal structures. In the region of $x \leqslant 2.8$, the main phase is $\mathrm{Gd}(\mathrm{Co}, \mathrm{Si})_{11}$, with a bodycentred tetragonal $\mathrm{BaCd}_{11}$-type structure (space group $\mathrm{I}_{1} /$ amd) with some impurity phases. For $x \geqslant 3.0$ the pattern of body-centred tetragonal $\mathrm{LaFe}_{9} \mathrm{Si}_{4}$-type structure [15] (space group $I 4 / \mathrm{mcm}$ ) appears, and up to $x=3.6$ it is superimposed on the pattern of the $\mathrm{BaCd}_{11}$ structure type. In fact, in the region $3.0 \leqslant x<3.8$, the $1: 11$ phase decreases gradually with increasing $x$, and vice versa the $1: 13$ phase becomes dominant. Finally, in the range $3.8 \leqslant x \leqslant 4.2$, almost single-phase samples of $\mathrm{LaFe}_{9} \mathrm{Si}_{4}$-type are formed with traces of $\mathrm{Gd}_{2} \mathrm{O}_{3}$ and the solid solution $\mathrm{Co}_{0.92} \mathrm{Si}_{0.08}$. The latter is ferromagnetic with $T_{\mathrm{C}} \sim 1000 \mathrm{~K}$ and, thus, adds an over-proportional contribution to the intrinsic paramagnetic high-temperature susceptibility of $\mathrm{GdCo}_{9-\delta} \mathrm{Si}_{4+\delta}$ samples, even in those with less than $2 \%$ impurity phase. In order to inhibit the formation of the ferromagnetic $\mathrm{Co}_{0.92} \mathrm{Si}_{0.08}$ phase we varied the nominal starting composition to $\mathrm{Gd}_{1.02} \mathrm{Co}_{9-\delta} \mathrm{Si}_{4+\delta}$ and obtained samples practically without any $\mathrm{Co}_{0.92} \mathrm{Si}_{0.08}$ impurity phase. A sample with the nominal composition $\mathrm{Gd}_{1.02} \mathrm{Co}_{9} \mathrm{Si}_{4}$ has, thus, been used for the magnetization studies presented below. The analysis of the $\mathrm{x}$-ray pattern of the latter revealed traces of $\mathrm{Gd}_{2} \mathrm{O}_{3}$ impurities, however, yielding negligible contributions to the magnetization data. XRD patterns of the composition range $3.6 \leqslant x \leqslant 4.0$ have been presented earlier in [14]; selected XRD 


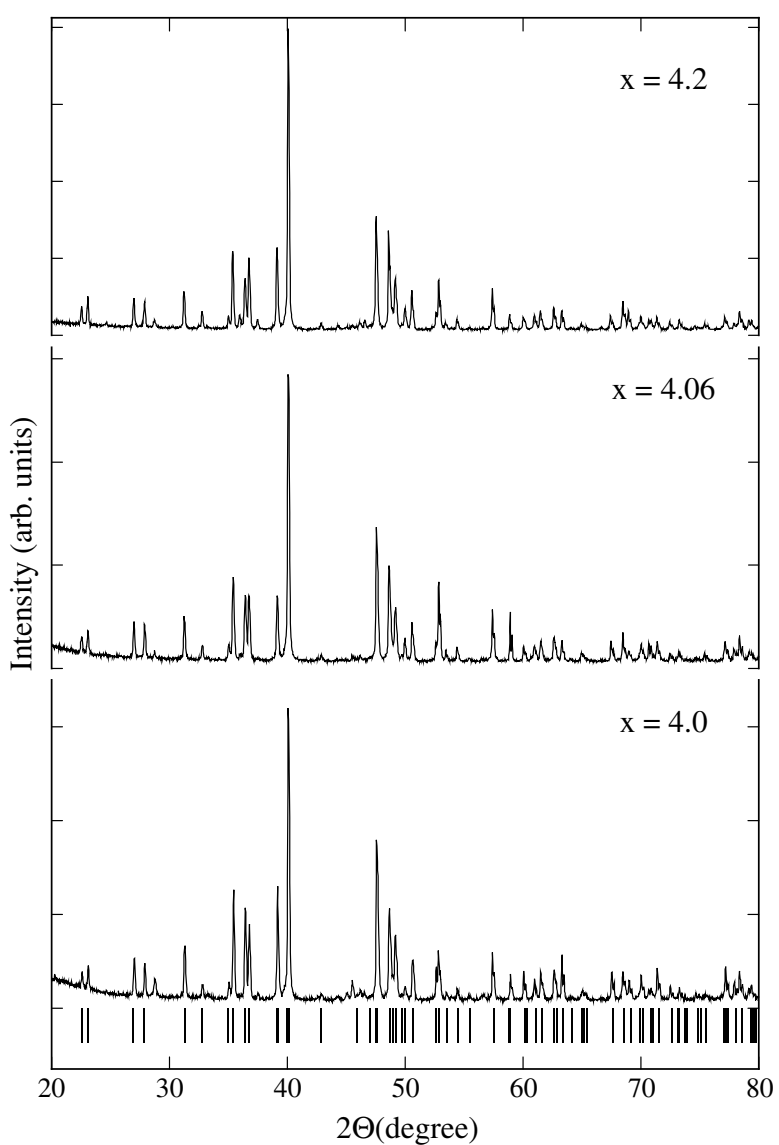

Figure 2. X-ray diffraction patterns for selected 1:13 type $\mathrm{GdCo}_{13-x} \mathrm{Si}_{x}$ samples; the calculated positions of the $\mathrm{x}$-ray reflections of $\mathrm{GdCo}_{9} \mathrm{Si}_{4}$ are indicated at the bottom.

Table 2. Lattice parameters of 1:13 type $\mathrm{GdCo}_{13-x} \mathrm{Si}_{x}$ samples.

\begin{tabular}{lllll}
\hline$x$ & $a(\AA)$ & $c(\AA)$ & $V\left(\AA^{3}\right)$ & $(c / a)$ \\
\hline 3.8 & $7.7745(6)$ & $11.493(1)$ & 694.67 & 1.478 \\
4.0 & $7.7702(5)$ & $11.499(1)$ & 694.25 & 1.480 \\
4.06 & $7.7685(5)$ & $11.503(1)$ & 693.32 & 1.481 \\
4.1 & $7.7698(6)$ & $11.502(1)$ & 694.39 & 1.480 \\
4.2 & $7.7643(5)$ & $11.506(1)$ & 693.63 & 1.482 \\
\hline
\end{tabular}

patterns for $4.0 \leqslant x \leqslant 4.2$ are shown in figure 2 . The lattice parameters of single-phase $\mathrm{Gd}(\mathrm{Co}, \mathrm{Si})_{13}$ are given in table 2. It is clearly seen that the substitution of Co by $\mathrm{Si}$ causes a shrinkage of the $a$ lattice parameter and a slight increase of the $c$ lattice parameter with a small total shrinkage in volume. These results are in agreement with the trends reported for $\mathrm{GdCo}_{9} \mathrm{Si}_{4}$ and $\mathrm{GdCo}_{9.5} \mathrm{Si}_{3.5}$ by Huang et al [9]. All technical details of the refinements were given earlier in context with our structure and microstructure investigation of $\mathrm{GdCo}_{13-x} \mathrm{Si}_{x}$ $(3.8 \leqslant x \leqslant 4.0)[14]$.

In the case of $\mathrm{LaCo}_{9} \mathrm{Si}_{4}$ and $\mathrm{YCo}_{9} \mathrm{Si}_{4}$ a fully ordered distribution of $\mathrm{La} / \mathrm{Y}, \mathrm{Co}$ and $\mathrm{Si}$ atoms within the space group $I 4 / \mathrm{mcm}$ has been revealed by single-crystal X-ray diffraction $[5,6]$ which is also corroborated by the concentration dependence of the residual resistivity in the solid solution $\mathrm{LaCo}_{13-x} \mathrm{Si}_{x}$ with a clear minimum at $x=4$ [7]. By analogy, we also expect 

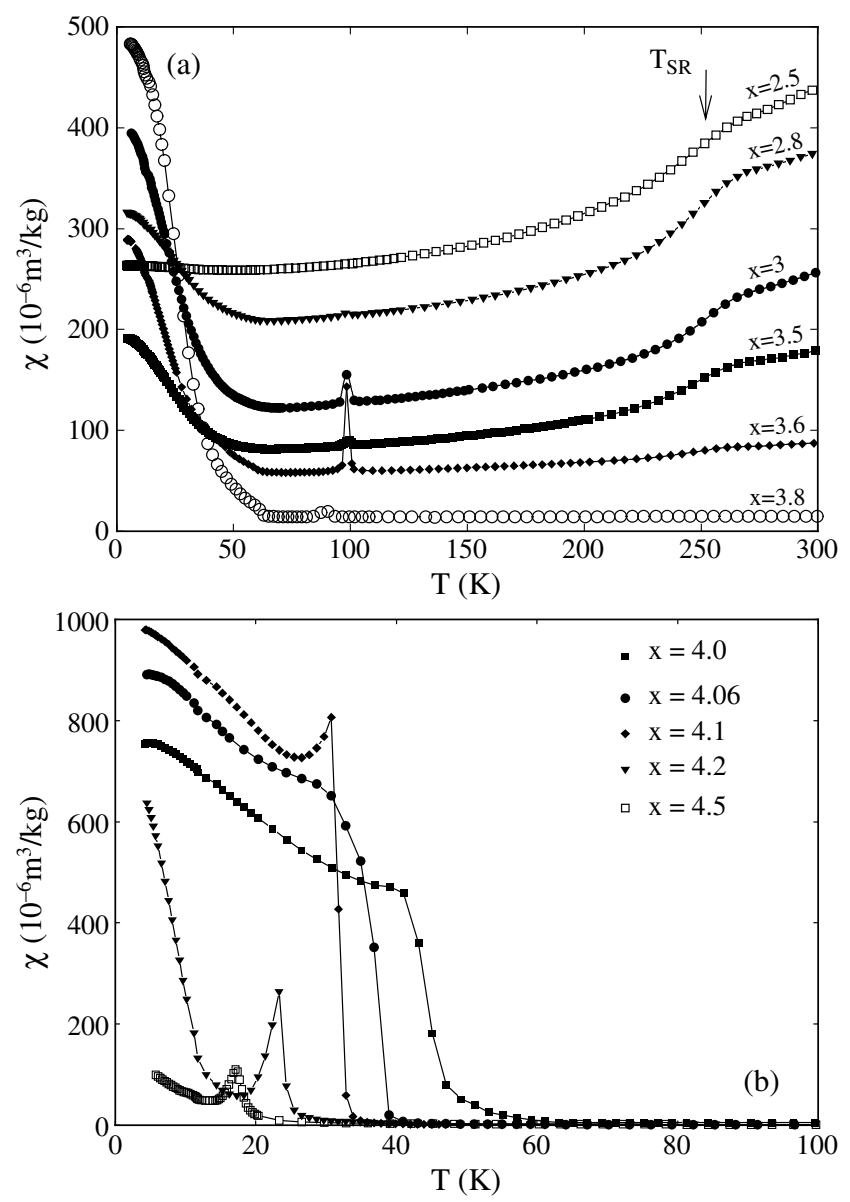

Figure 3. (a) Temperature dependent in phase ac susceptibility $\chi(T)$ of $\mathrm{GdCo}_{13-x} \mathrm{Si}_{x}$ samples with $2.5 \leqslant x \leqslant 3.8 ; T_{\mathrm{SR}}$ indicates the spin-reorientation transition of the $\mathrm{GdCo} \sim 9 \mathrm{Si}_{\sim 2}$ phase; (b) the in phase ac susceptibility $\chi(T)$ of $\mathrm{GdCo}_{13-x} \mathrm{Si}_{x}$ compounds with $4.0 \leqslant x \leqslant 4.5$.

a fully ordered distribution of the atoms for $\mathrm{GdCo}_{9} \mathrm{Si}_{4}$, which is in fact well supported by the relatively low residual resistivity (see below) of stoichiometric $\mathrm{GdCo}_{9} \mathrm{Si}_{4}$.

\subsection{Magnetic characterization of $\mathrm{GdCo}_{13-x} \mathrm{Si}_{x}$ alloys}

The temperature dependence of the real (in phase) components of the complex magnetic ac susceptibility $\chi(T)$ of $\mathrm{GdCo}_{13-x} \mathrm{Si}_{x}$ is presented in figures 3(a) and (b). The imaginary components have been omitted for clarity-they are negligible in the paramagnetic regime and $\leqslant 0.1$ times the real part in the magnetically ordered state. The alloys with $x=2.5-3.8$, see figure 3(a), exhibit magnetic phases with $T_{\mathrm{C}}$ above room temperature. The dominant one is obviously due to $\mathrm{Gd}(\mathrm{Co}, \mathrm{Si})_{11}$, because the susceptibility anomalies at about $260 \mathrm{~K}$ match with the spin re-orientation transition of ferrimagnetic $\mathrm{GdCo}_{9} \mathrm{Si}_{2}$ reported in [16]. As expected from the above EMPA and XRD results, the $\mathrm{Gd}(\mathrm{Co}, \mathrm{Si})_{11}$ phase and thus the spin re-orientation transition at $260 \mathrm{~K}$ disappears upon increasing the Si content up to $x=3.8$. Concomitantly with the decreasing $\mathrm{Gd}(\mathrm{Co}, \mathrm{Si})_{11}$ contribution to the ac susceptibility, there is an increasing 
contribution with a magnetic anomaly at or below $60 \mathrm{~K}$ which is attributed to the $\mathrm{GdCo}_{9-\delta} \mathrm{Si}_{4+\delta}$ phase. In the two-phase region $2.8 \leqslant x \leqslant 3.6$, the susceptibility data exhibit additional small peaks around $100 \mathrm{~K}$ which might be due to an additional secondary phase not resolved by EMPA and XRD. $\mathrm{GdCo}_{9.2} \mathrm{Si}_{3.8}$ appears to be free from $\mathrm{Gd}(\mathrm{Co}, \mathrm{Si})_{11}$ phase, but still exhibits a relatively high room temperature susceptibility which is due to ferromagnetic $\mathrm{Co}_{0.92} \mathrm{Si}_{0.08}$ impurities. Remarkably sharp magnetic transitions are shown in figure 3(b) for $\mathrm{GdCo}_{9-\delta} \mathrm{Si}_{4+\delta}$, which according to EMPA and XRD is obtained as almost single-phase material from the alloys $\mathrm{GdCo}_{13-x} \mathrm{Si}_{x}$ with $4.0 \leqslant x \leqslant 4.2$. Within the composition range $x=3.8-4.5$ there is a monotonic decrease of the magnetic ordering temperatures, $T_{\mathrm{C}}$, from about $60 \mathrm{~K}$ at $x=3.8$ (estimated from the initial increase of the ac susceptibility shown in figure 3(a)) to $28 \mathrm{~K}$ for $x=4.2$ (single-phase sample) and to $18 \mathrm{~K}$ for $x=4.5$ (multi-phase sample).

For $2.8 \leqslant x \leqslant 3.8$, where EMPA yields a roughly constant composition for the $\mathrm{GdCo}_{9-\delta} \mathrm{Si}_{4+\delta}$ phase with $\delta \sim-0.2$, we observe practically the same magnetic anomaly with $T_{\mathrm{C}} \sim 60 \mathrm{~K}$ and a gradual increase of the change of the susceptibility below $60 \mathrm{~K}$, i.e. $\chi(4.2 \mathrm{~K})-\chi(60 \mathrm{~K})$ as expected from the fraction of the 1-9-4 phase according to table 1 . The susceptibility data thus corroborate the rather narrow homogeneity range of the $\mathrm{GdCo}_{9-\delta} \mathrm{Si}_{4+\delta}$ phase with $-0.2 \leqslant \delta \leqslant 0.2$. For other $\mathrm{RCo}_{9+\delta} \mathrm{Si}_{4-\delta}$ with larger rare earth ions the homogeneity range is wider, in particular for $\mathrm{LaCo}_{13-x} \mathrm{Si}_{x}$ with the tetragonal $\mathrm{LaFe}_{9} \mathrm{Si}_{4}$ type structure in between $x=2-5$ and cubic $\mathrm{NaZn}_{13}$-type structure up to binary $\mathrm{LaCo}_{13}$, i.e. the end of the solid solution $\mathrm{La}(\mathrm{Co}, \mathrm{Si})_{13}[17]$.

\subsection{Magnetic properties of $\mathrm{GdCo}_{9} \mathrm{Si}_{4}$}

The ac susceptibility in figure 3(b) reveals for single-phase 1:13 type $\mathrm{GdCo}_{9} \mathrm{Si}_{4}$ a rather sharp magnetic anomaly at about $47 \mathrm{~K}$. The nature of magnetism has been studied by temperature dependent magnetization measurements, $M(T)$, and isothermal magnetization measurements, $M(H)$, in static magnetic fields up to $6 \mathrm{~T}$ using a SQUID magnetometer and in pulsed magnetic fields up to $48 \mathrm{~T}$. The temperature dependent magnetization data, $M(T)$, measured in fields of $0.1,1$ and $3 \mathrm{~T}$ are shown in figure 4 . The inverse susceptibility $1 / \chi(T)$ shown in the inset of figure 4 reveals Curie-Weiss-like paramagnetic behaviour above about $80 \mathrm{~K}$ where the 1 and $3 \mathrm{~T}$ susceptibilities match rather well; just the $0.1 \mathrm{~T}$ data slightly deviate by some additional temperature independent contribution which is most likely due to tiny amounts of magnetic impurities. The usual modified Curie-Weiss fit of the form $\chi=C /\left(T-\Theta_{\mathrm{p}}\right)+\chi_{0}$ applied to the temperature interval $100-300 \mathrm{~K}$ yields a Curie constant, $C=8.5$ (2) emu K mol ${ }^{-1}$, a paramagnetic Curie temperature $\Theta_{\mathrm{p}}=-17(1) \mathrm{K}$ and a temperature independent Pauli susceptibility $\chi_{0} \simeq 2.0 \times 10^{-3} \mathrm{emu} \mathrm{mol}^{-1}$. The Curie constant yields an effective paramagnetic moment, $\mu_{\text {eff }}=8.2$ (1) $\mu_{\mathrm{B}} /$ f.u., which is larger than the usual values expected for gadolinium $\left(\sim 7.9 \mu_{\mathrm{B}}\right)$, i.e. Gd and Co moments are both contributing to the effective paramagnetic moment. The corresponding fit applied to the paramagnetic reference compound $\mathrm{LaCo}_{9} \mathrm{Si}_{4}$ in the same temperature interval yields $C \simeq 1.6 \mathrm{emu} \mathrm{K} \mathrm{mol}{ }^{-1}, \Theta_{\mathrm{p}} \simeq 28 \mathrm{~K}$ and $\chi_{0} \simeq$ $2 \times 10^{-3} \mathrm{emu} \mathrm{mol}^{-1}$, i.e. the strongly exchange enhanced Pauli paramagnetic behaviour of the Co $3 \mathrm{~d}$ bands (dominated by the 16k sites [5]) contributes to an effective paramagnetic moment and simultaneously to an enhanced temperature independent Pauli susceptibility with comparable magnitude in $\mathrm{GdCo}_{9} \mathrm{Si}_{4}$ and $\mathrm{LaCo}_{9} \mathrm{Si}_{4}$. The isothermal magnetization, $M(H)$ of $\mathrm{GdCo}_{9} \mathrm{Si}_{4}$ shown in figure 5, reveals on the other hand at $2.5 \mathrm{~K}$ and $6 \mathrm{~T}$ a saturation magnetization of $5.0 \mu_{\mathrm{B}} /$ f.u. (i.e. $35 \mathrm{emu} \mathrm{g}^{-1}$ in agreement with [9]), which is well below the $7 \mu_{\mathrm{B}} /$ f.u. (i.e. $49 \mathrm{emu} \mathrm{g}^{-1}$ ) expected for a fully polarized Gd sublattice. The reduction of the low temperature saturation magnetization of $\mathrm{GdCo}_{9} \mathrm{Si}_{4}$ by about $2 \mu_{\mathrm{B}} / \mathrm{f}$.u. is similar to the low temperature saturation magnetization of the itinerant electron metamagnet $\mathrm{LaCo}_{9} \mathrm{Si}_{4}$ with 


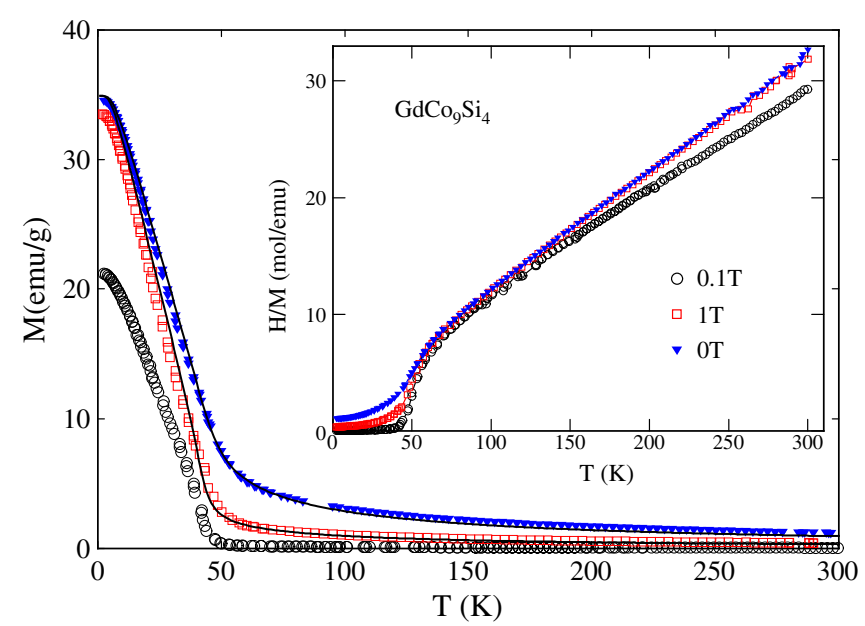

Figure 4. Temperature dependent magnetization, $M(T)$, of $\mathrm{GdCo}_{9} \mathrm{Si}_{4}$ measured in various external fields as labelled; solid lines are numerical results of the Néel theory (see text); inset: the corresponding inverse susceptibility, $H / M(T)$.

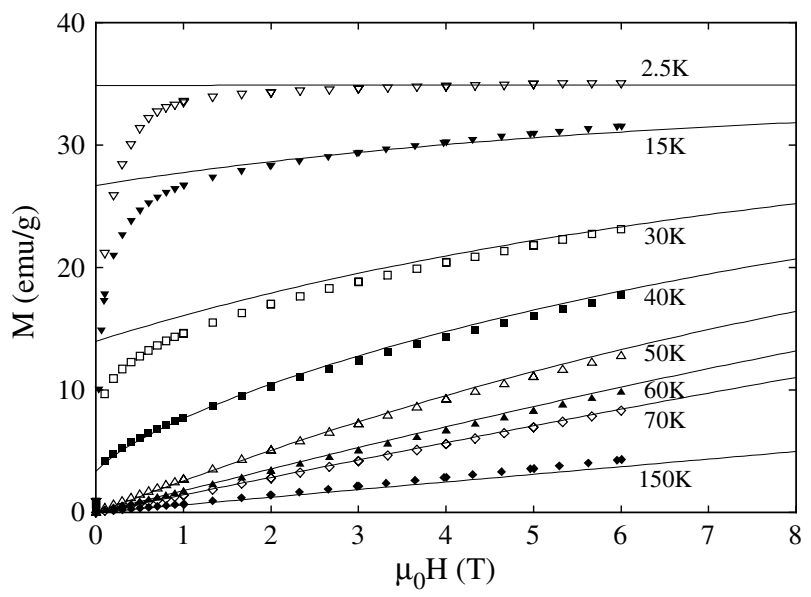

Figure 5. Isothermal magnetization data $M(H)$ of $\mathrm{GdCo}_{9} \mathrm{Si}_{4}$; the solid lines are according to the Néel model; see the text.

$1.6 \mu_{\mathrm{B}} /$ f.u. at $2 \mathrm{~K}$ and $15 \mathrm{~T}$ and also that of the isoelectronic and isostructural weak itinerant ferromagnet $\mathrm{YCo}_{9} \mathrm{Si}_{4}$ with $M_{\text {sat }} \simeq 2 \mu_{\mathrm{B}} /$ f.u. (see [6]). This refers to a ferrimagnetic coupling of the magnetic moments on the $\mathrm{Gd}$ and Co sublattices.

A simple model which is suited to analyse the $M(T)$ and $M(H)$ data in order to obtain an estimate for the strength of the inter- and intra-sublattice exchange coupling of two-sublattice collinear ferrimagnets is the Néel theory (see $[18,19]$ ) based on the local moment molecular field model. This approach is well justified for the Gd magnetism and of course limited with respect to the Co magnetism because of the dual itinerant and local nature of the latter, but nevertheless useful to analyse the magnetization data of $\mathrm{GdCo}_{9} \mathrm{Si}_{4}$ with a minimum set of parameters.

According to the Néel model, sublattice magnetization $M_{\mathrm{Gd}}$ and $M_{\mathrm{Co}}$ are given by

$$
M^{\mathrm{Gd}}=M_{0}^{\mathrm{Gd}} B_{S_{\mathrm{Gd}}}\left(\frac{g_{\mathrm{Gd}} \mu_{\mathrm{B}} S_{\mathrm{Gd}} H_{\mathrm{Gd}}}{k_{\mathrm{B}} T}\right)
$$




$$
M^{\mathrm{Co}}=M_{0}^{\mathrm{Co}} B_{S_{\mathrm{Co}}}\left(\frac{g_{\mathrm{Co}} \mu_{\mathrm{B}} S_{\mathrm{Co}} H_{\mathrm{Co}}}{k_{\mathrm{B}} T}\right),
$$

where $M_{0}^{\mathrm{Gd}}$, the low temperature saturation magnetization of the Gd sublattice, with the $4 \mathrm{f}$ being in a ${ }^{8} \mathrm{~S}_{7 / 2}$ state, is $7 \mu_{\mathrm{B}} /$ f.u., and $M_{0}^{\text {Co }}$ is defined by the difference between $M_{0}^{\mathrm{Gd}}$ and the experimental low temperature saturation magnetization $M(6 \mathrm{~T}) \simeq 35 \mathrm{emu} \mathrm{g}^{-1}=5.0 \mu_{\mathrm{B}} / \mathrm{f}$.u., yielding $M_{0}^{\mathrm{Co}}=2 \mu_{\mathrm{B}} /$ f.u. for $\mathrm{GdCo}_{9} \mathrm{Si}_{4} . B_{S}$ is the Brillouin function, $S_{\mathrm{Gd}}=7 / 2$ and $g_{\mathrm{Gd}}=2$ the Landé factor of the $\mathrm{Gd} 4 \mathrm{f}^{7}$ shell. We take $S_{\mathrm{Co}}=1 / 2$ and $g_{\mathrm{Co}}=1$ for the $16 \mathrm{k}$ Co sites (4 at./f.u.) and no moment for the others, since there are five almost non-magnetic Co/f.u. on the 161 and $4 \mathrm{~d}$ sites (see section 1 and $[5,6]$ ). This yields $M_{0}^{\mathrm{Co}}=2 \mu_{\mathrm{B}} /$ f.u., which is suited to satisfy the experimental results: $M_{0} \simeq 5 \mu_{\mathrm{B}} /$ f.u. $=M_{0}^{\mathrm{Gd}}-M_{0}^{\mathrm{Co}}$. The artificially reduced $g$-factor is the consequence of applying a local-moment model to moments with dual itinerant and local nature. $H_{\mathrm{Gd}}$ and $H_{\mathrm{Co}}$ are the effective fields acting on the $\mathrm{Gd}$ and Co moments, respectively, given by

$$
\begin{aligned}
& H_{\mathrm{Gd}}=H_{0}+\gamma_{\mathrm{GdGd}} M^{\mathrm{Gd}}+\gamma_{\mathrm{GdCo}} M^{\mathrm{Co}} \\
& H_{\mathrm{Co}}=H_{0}+\gamma_{\mathrm{GdCo}} M^{\mathrm{Gd}}+\gamma_{\mathrm{CoCo}} M^{\mathrm{Co}},
\end{aligned}
$$

with the external magnetic field, $H_{0}$, two intra-sublattice molecular field coefficients $\gamma_{\mathrm{GdGd}}$ and $\gamma_{\mathrm{CoCo}}$ and one inter-sublattice molecular field coefficient $\gamma_{\mathrm{GdCo}}$. Equations (1) and (2) are, thus, a coupled set of transcendent equations which are solved by numerical methods.

The most reasonable description of $M(T)$ in the paramagnetic regime (see figure 4) and of $M(T, H)$ measured in static fields up to $6 \mathrm{~T}$ as shown in figure 5 is achieved with a set of molecular field coefficients $\gamma_{\mathrm{CoCo}}=90 \mathrm{G} \mathrm{mol} \mathrm{emu}^{-1}, \gamma_{\mathrm{GdCo}}=-9.8 \mathrm{G} \mathrm{mol} \mathrm{emu}^{-1}$ and $\gamma_{\mathrm{GdGd}}=0.03 \mathrm{G} \mathrm{mol} \mathrm{emu}{ }^{-1}$. Accordingly, the strongest exchange coupling appears within the sublattice of $16 \mathrm{k}$ Co sites, the ferrimagnetic inter-sublattice coupling is one order of magnitude smaller and there is a rather insignificant exchange coupling within the Gd subsystem $\left(\gamma_{\mathrm{GdGd}}=\right.$ $0.03 \mathrm{G} \mathrm{mol} \mathrm{emu}^{-1}$ accounts for the dipolar interaction of the $\mathrm{Gd}$ moments). The results of the Néel model are plotted in figures 4 and 5 by solid lines, which agree reasonably well in the paramagnetic state and above about $1 \mathrm{~T}$ in the ordered state.

Figure 6 shows $M(H)$ measurements up to $47 \mathrm{~T}$ performed on $\mathrm{GdCo}_{9} \mathrm{Si}_{4}$ at temperatures $T=4.2-30 \mathrm{~K}$. The $M(H)$ curves reveal a breaking up of the collinear ferrimagnetic coupling of the Gd and Co subsystems towards a non-collinear magnetic state at a lower critical field $\mu_{0} H_{1} \simeq 27 \mathrm{~T}$ in perfect agreement with the value expected according to Clark and Callen [20] for a Néel ferrimagnet with weak anisotropy in high magnetic fields. In this model, the lower critical field is given by $\mu_{0} H_{\mathrm{l}}(T=0)=\left|\gamma_{\mathrm{GdCo}}\left(M_{0}^{\mathrm{Gd}}-M_{0}^{\mathrm{Co}}\right)\right|$, yielding $27.4 \mathrm{~T}$ with the above values, $M_{0}^{\mathrm{Gd}}=49 \mathrm{emu} \mathrm{g}^{-1}$ and $M_{0}^{\mathrm{Co}}=14 \mathrm{emu} \mathrm{g}^{-1}$, and the above inter-sublattice molecular field coefficient $\gamma_{\mathrm{GdCo}}=-9.8 \mathrm{G} \mathrm{mol} \mathrm{emu}^{-1}$. The upper critical field, $\mu_{0} H_{\mathrm{u}}$, is observed at about $44 \mathrm{~T}$, where the metamagnetic re-orientation from the ferrimagnetic via a non-collinear magnetic state to a collinear ferromagnetic alignment of the Gd and Co moments is completed. This value of $44 \mathrm{~T}$ is slightly lower than expected from the molecular field model [20], yielding $\mu_{0} H_{\mathrm{u}}=\gamma_{\mathrm{GdCo}}\left(M_{0}^{\mathrm{Gd}}+M_{0}^{\mathrm{Co}}\right) \simeq 49.4 \mathrm{~T}$. The total magnetization, $M_{\text {sat }} \simeq 8.7(0.3) \mu_{\mathrm{B}} /$ f.u., measured at the highest field at $4.2 \mathrm{~K}$ corresponds to the sum of the two sublattice magnetizations $M_{0}^{\mathrm{Gd}}+M_{0}^{\mathrm{Co}}$ and agrees reasonably well with the above values $M_{0}^{\mathrm{Gd}}$ and $M_{0}^{\mathrm{Co}}$ calculated from low field measurements, yielding $9.0 \mu_{\mathrm{B}} /$ f.u. in total. The slope of the magnetization between the lower and the upper critical field (extrapolating through the origin) corresponds for $T \ll T_{\mathrm{C}}$ to $\left|1 / \gamma_{\mathrm{GdCo}}\right|$ and at $4.2 \mathrm{~K}$ yields $\gamma_{\mathrm{GdCo}} \simeq-9.2 \mathrm{G} \mathrm{mol} \mathrm{emu}^{-1}$, which is in reasonable agreement with the above value, $-9.8 \mathrm{G} \mathrm{mol} \mathrm{emu}^{-1}$, estimated from the $M(T)$ data in the paramagnetic regime (see figure 4) and the low field $M(T, H)$ data in figure 5. Also the reduction of $H_{1}$ and $H_{\mathrm{u}}$ as indicated by the comparison of $M(H)$ at 4.2 and $15 \mathrm{~K}$ in figure 6 agrees with that expected from the molecular field model. The Néel model, 

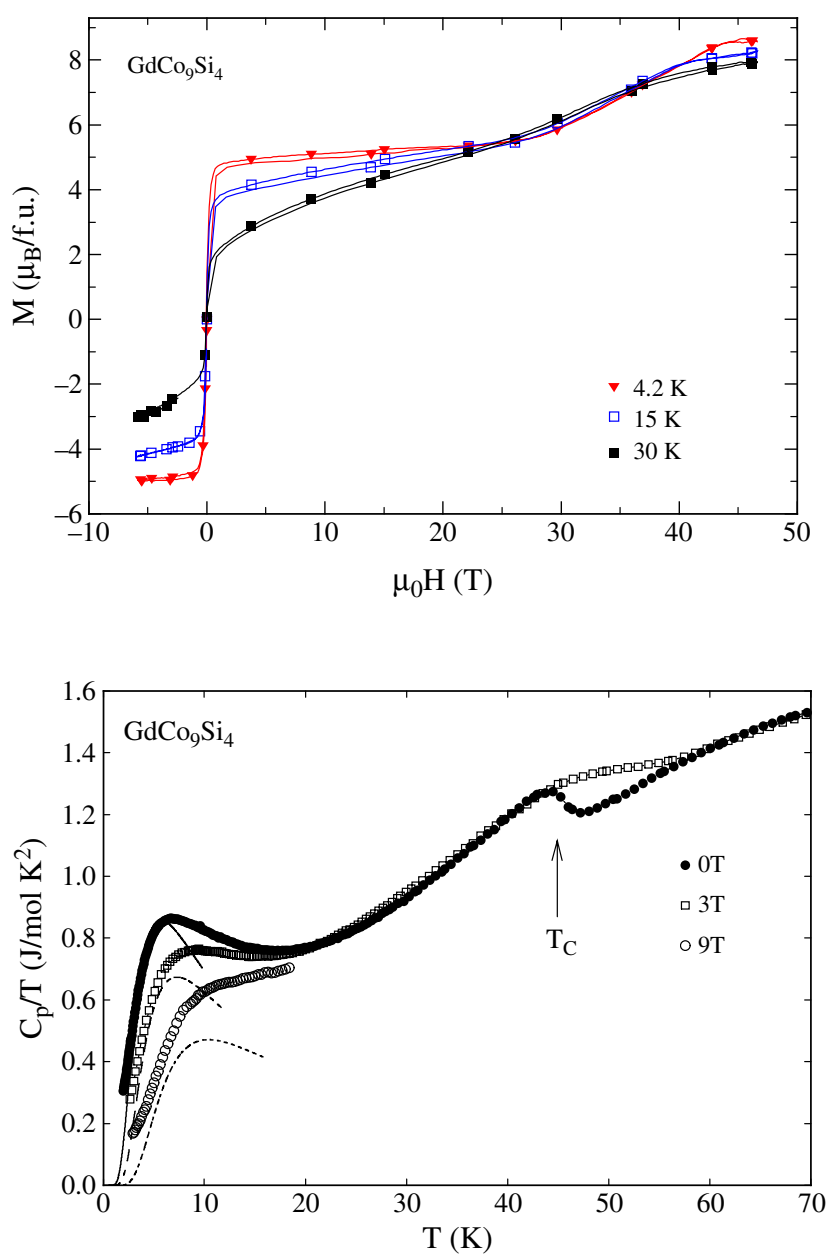

Figure 6. High field measurements of the isothermal magnetization $M(H)$ of $\mathrm{GdCo}_{9} \mathrm{Si}_{4}$ at $4.2,15$ and $30 \mathrm{~K}$ and as labelled. Data points are represented by lines and only a few selected points additionally by symbols.

Figure 7. Temperature dependence of the specific heat $C_{\mathrm{p}} / T$ for $\mathrm{GdCo}_{9} \mathrm{Si}_{4}$ with external applied fields up to $9 \mathrm{~T}$; the solid and dashed lines are calculated Zeeman contributions as explained in the text.

thus, provides a reasonable quantitative description of the ferrimagnetism in $\mathrm{GdCo}_{9} \mathrm{Si}_{4}$, even though the magnetism on the Co sublattice has been accounted for in the simplest way possible.

The specific heat of $\mathrm{GdCo}_{9} \mathrm{Si}_{4}$ shown in figure 7 as $C / T$ versus $T$ reveals a rather sharp anomaly associated with the magnetic phase transition at $T_{\mathrm{C}} \simeq 47 \mathrm{~K}$. The specific heat jump related to this anomaly, $\Delta C \simeq 8.5 \mathrm{~J} \mathrm{~mol}^{-1} \mathrm{~K}^{-1}$, is significantly smaller than that expected for a mean-field-like phase transition of the Gd subsystem, i.e. $\Delta C \sim 20.15 \mathrm{~J} \mathrm{~mol}^{-1} \mathrm{~K}^{-1}$ for the $J=7 / 24$ f moments of Gd. However, the normalized jump $\Delta C / T_{\mathrm{C}} \sim 180 \mathrm{~mJ} \mathrm{~mol}^{-1} \mathrm{~K}^{-2}$ is of the same order of magnitude as the anomaly of $\mathrm{YCo}_{9} \mathrm{Si}_{4}\left(\Delta C / T_{\mathrm{C}} \sim 100 \mathrm{~mJ} \mathrm{~mol}^{-1} \mathrm{~K}^{-2}\right)[6]$ and is, thus, indicative of the dual itinerant and localized nature of the Co magnetism. The similar magnitude of the specific heat anomalies of $\mathrm{YCo}_{9} \mathrm{Si}_{4}$ and $\mathrm{GdCo}_{9} \mathrm{Si}_{4}$ corroborates the above conclusion that the magnetic phase transition is primarily driven by the Co $3 \mathrm{~d}$ bands. The low temperature Schottky anomaly with a maximum in $C / T$ versus $T$ (at about $6 \mathrm{~K}$ in zero external field) further corroborates the Néel model, which assumes an effective internal field acting on the Gd sublattice, $H_{\mathrm{Gd}} \simeq H_{0}+\gamma_{\mathrm{GdCo}} M_{0}^{\mathrm{Co}} \simeq H_{0}+11$ T. thus implying a Zeeman splitting of the Gd $S=7 / 2$ states yielding specific heat contributions as indicated by the solid and dashed lines in figure 7 , showing calculations for $11 \mathrm{~T}\left(H_{0}=0 \mathrm{~T}\right), 14 \mathrm{~T}\left(H_{0}=3 \mathrm{~T}\right)$ and $20 \mathrm{~T}\left(H_{0}=9 \mathrm{~T}\right)$. These Zeeman contributions agree with the experimental data rather well at 


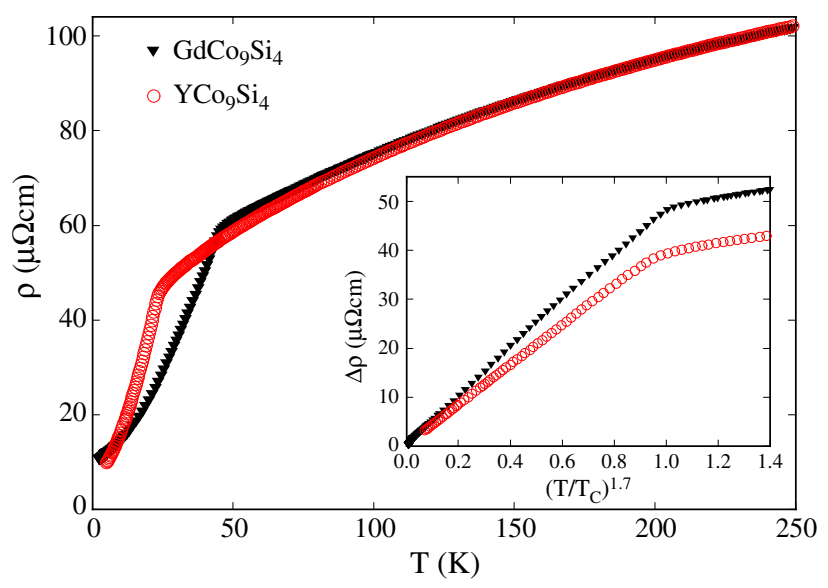

Figure 8. Temperature dependent resistivity $\rho(T)$ of $\mathrm{GdCo}_{9} \mathrm{Si}_{4}$ in comparison with $\mathrm{YCo}_{9} \mathrm{Si}_{4}$. The inset shows the resistivity versus normalized temperature.

low temperatures and deviate with increasing temperature because of phonon contributions and contributions from the Co $3 \mathrm{~d}$ magnetism.

The temperature dependent resistivity, $\rho(T)$, of $\mathrm{GdCo}_{9} \mathrm{Si}_{4}$, shown in figure 8 , is well described in the ferrimagnetic state $\left(T<T_{\mathrm{C}} / 2\right)$ by a power law behaviour $\rho(T)=\rho_{0}+A T^{\alpha}$, with a residual resistivity $\rho_{0}=10.4 \mu \Omega \mathrm{cm}, A=0.08 \mu \Omega \mathrm{cm} \mathrm{K}^{-\alpha}$ and an exponent $\alpha=1.7$. The corresponding fit for the weak itinerant ferromagnet $\mathrm{YCo}_{9} \mathrm{Si}_{4}$ yields $\rho_{0}=7 \mu \Omega \mathrm{cm}$, $A=0.176 \mu \Omega \mathrm{cm} \mathrm{K}^{-\alpha}$ and $\alpha=1.72$ [6]. The results indicate that the coefficient $A$ scales with $T_{\mathrm{C}}$, i.e. $A / T_{\mathrm{C}}{ }^{\alpha}$ is similar for both compounds. The resistivity versus normalized temperature, $\left(T / T_{\mathrm{C}}\right)^{1.7}$, is shown in the inset of figure 8. Similar results with a clear deviation from the usual $T^{2}$ Fermi liquid behaviour have been reported by Steiner et al [21] for the weak itinerant ferromagnets $\mathrm{YNi}_{3}$ and $\mathrm{Ni}_{3} \mathrm{Al}$ which exhibit non-Fermi-liquid- (NFL-) like low temperature resistivity, $\rho(T)=\rho_{0}+A T^{\sim 1.65}$.

The observed $\rho(T)$ of $\mathrm{GdCo}_{9} \mathrm{Si}_{4}$ and $\mathrm{YCo}_{9} \mathrm{Si}_{4}$ exhibiting the same qualitative behaviour with an NFL exponent $\alpha \simeq 1.7$ indicates that the magnetic scattering by the $\mathrm{d}$ subsystem dominates while scattering by the Gd 4 f moments is weak. The negative curvature of $\rho(T)$ in the paramagnetic state is a typical feature of systems with significant spin fluctuations. Scattering of conduction electrons on the localized Gd ions in the paramagnetic temperature range does not cause any additional temperature dependence since crystal field effects are negligible for the ${ }^{8} \mathrm{~S}_{7 / 2} 4 \mathrm{f}$-states.

\section{Electronic structure calculation}

To investigate the magnetism of $\mathrm{GdCo}_{9} \mathrm{Si}_{4}$ on a microscopic basis, we carried out density functional electronic structure calculations. A full-potential non-orthogonal local-orbital (FPLO) calculation scheme [22] within the local spin density approximation (LSDA) was employed. In the scalar-relativistic calculations the exchange and correlation potential of Perdew and Wang [23] was used. All calculations were done for the experimental lattice constants $a=7.770, c=11.499 \AA$. As the basis set, Gd (4f, 5s, 5p, 6s, 6p, 5d), Co (3s, $3 \mathrm{p}, 4 \mathrm{~s}, 4 \mathrm{p}, 3 \mathrm{~d})$ and $\mathrm{Si}(3 \mathrm{~s}, 3 \mathrm{p}, 3 \mathrm{~d})$ states were employed. The lower lying states were treated fully relativistically as core states. The spatial extension of the basis orbitals, controlled by a confining potential $\left(r / r_{0}\right)^{4}$, was optimized to minimize the total energy [24]. A $k$-mesh of 1000 points in the Brillouin zone was used to ensure accurate band structure and total energy results. To account for the strong Coulomb correlations in the $\mathrm{Gd} 4 \mathrm{f}$ states, these states were treated within the so-called LSDA $+U$ (atomic limit) formalism with $U=8 \mathrm{eV}$. 
As result of the calculation, we find a spin-polarized ground state with a total magnetic moment of $3.53 \mu_{\mathrm{B}} /$ f.u. We ascribe the deviation to the experimental value $\left(5.0 \mu_{\mathrm{B}} / \mathrm{f}\right.$.u. $)$ to the strong spin fluctuations present in this system, similar to the situation in the itinerant ferromagnets $\mathrm{Na}(\mathrm{K}) \mathrm{Fe}_{4} \mathrm{Sb}_{12}$ [25]. The total magnetic moment consists essentially of contributions from the fully polarized $\operatorname{Gd} 4 \mathrm{f}\left(M_{\mathrm{Gd}-4 \mathrm{f}}=6.97 \mu_{\mathrm{B}} /\right.$ f.u. $)$ states and the antiparallel oriented Co 3d $\left(M_{\mathrm{Co}-3 \mathrm{~d}}=-3.92 \mu_{\mathrm{B}} /\right.$ f.u. $)$ moments. Besides the Gd $5 \mathrm{~d}$ states that have a small polarization $\left(M_{\mathrm{Gd}-5 \mathrm{~d}}=0.17 \mu_{\mathrm{B}} /\right.$ f.u. $)$ parallel to the $4 \mathrm{f}$ moments, the contribution of all other orbitals is negligibly small. For the site projected magnetic moments, we find $M_{\mathrm{tot}}^{\mathrm{Gd}}=7.2 \mu_{\mathrm{B}} /$ f.u. and $M_{\mathrm{tot}}^{\mathrm{Co}}=-3.85 \mu_{\mathrm{B}} /$ f.u. for the sum over all Co sites. The on-site contribution, representing the more localized part of the magnetic moments (not taking into account the overlap part that is mainly due to contributions from the interstitial region), amounts to $M_{o s}^{\mathrm{Gd}}=7.15 \mu_{\mathrm{B}} /$ f.u. and $M_{o s}^{\mathrm{Co}}=-4.46 \mu_{\mathrm{B}} /$ f.u.

Almost $80 \%$ of the Co moment can be assigned to the $16 \mathrm{k}$ Co sites, similar to the situation in $\mathrm{LaCo}_{9} \mathrm{Si}_{4}$ [5], although the absolute values of the calculated Co moments are about three times larger in the Gd compound. A posteriori, this justifies the approximation to consider $16 \mathrm{k}$ Co moments only for the calculation of the molecular field coefficients.

To evaluate the lower critical field $B_{1}$, which marks the onset of the linear increase of the magnetization, we applied a technique which can be described as a virtual metamagnetic approach [26]. This approach maps fixed-spin-moment (FSM) calculations on a collinearly restricted two-sublattice model with rigid Gd and Co sublattices. According to the result of the unrestricted calculation, the inter-sublattice exchange interaction leads to a ferrimagnetic ground state. A ferromagnetic configuration is stable in applied magnetic field parallel to the easy direction exceeding a critical value $B_{\mathrm{c}}=\gamma_{4 \mathrm{f}-3 \mathrm{~d}} M^{\mathrm{Gd}}$, because $M^{\mathrm{Gd}}>M^{\mathrm{Co}}$ in $\mathrm{GdCo}_{9} \mathrm{Si}_{4}$. For magnetic fields $B$ larger than $B_{\mathrm{c}}, B$ overcomes the exchange field generated by the sublattice with the larger moment (i.e. $M^{\mathrm{Gd}}$ ) on the sublattice with the smaller moment (i.e. $M^{\mathrm{Co}}$ ). Thus, under the restriction of collinear sublattices, the system would undergo a metamagnetic transition for a critical field $B_{\mathrm{c}}=\gamma_{4 \mathrm{f}-3 \mathrm{~d}} M^{\mathrm{Gd}}$. As a computational artifact due to the collinear constraint, this transition would be first order. However, the real, unrestricted system can develop a canted spin structure and does not need to show a metamagnetic behaviour. In mean field theory [20] the onset of this non-collinear state can be induced by a lower critical field $B_{1}=\gamma_{4 \mathrm{f}-3 \mathrm{~d}}\left|M^{\mathrm{Gd}}-M^{\mathrm{Co}}\right|$. Thus, we find $B_{1}=\left[\left(M^{\mathrm{Gd}}-M^{\mathrm{Co}}\right) / M^{\mathrm{Gd}}\right] B_{\mathrm{c}}$.

In order to evaluate the lower critical field $B_{1}$, we calculated the critical field $B_{\mathrm{c}}$ from the total energy of the fixed spin moment curve. Figure 9 shows the total energy of $\mathrm{GdCo}_{9} \mathrm{Si}_{4}$ as a function of the total spin moment. The critical field $B_{\mathrm{c}}$ can be estimated from the slope $\mathrm{d} E / \mathrm{d} M$ of the common tangent (Helmholtz construction) between the local minima for the ferrimagnetic and the ferromagnetic state. According to figure 9, we find the slope $\mathrm{d} E / \mathrm{d} M=4.16 \mathrm{meV} / \mu_{\mathrm{B}}$ corresponding to $71.7 \mathrm{~T}$.

Independent from the two-sublattice model, the calculation of the critical field $B_{\mathrm{c}}$ by the fixed spin moment technique does not rely on any specific assignment of individual magnetic moments to the different kinds of atoms. However, for the evaluation of the lower critical field $B_{1}$ marking the onset of a canted spin structure, an assignment of the individual sublattice contribution is required. Because such an assignment is not unique, this results in a small ambiguity depending on the chosen projection method. Thus, we find $B_{1}=27.3 \mathrm{~T}$ for the above mentioned on-site magnetic moments and $B_{1}=33.4 \mathrm{~T}$ for the magnetic moments including the overlap contributions. Considering the limitations of the two-sublattice model for the case of the ternary $\mathrm{GdCo}_{9} \mathrm{Si}_{4}$ compound, these values are in surprisingly good agreement with the experimental value of $27 \mathrm{~T}$. It is well known that the LSDA has difficulties in describing the presence of strong spin fluctuations as in $\mathrm{GdCo}_{9} \mathrm{Si}_{4}$. The surprisingly good agreement presumably originates from a cancellation of two errors: (i) for the magnetic moment 


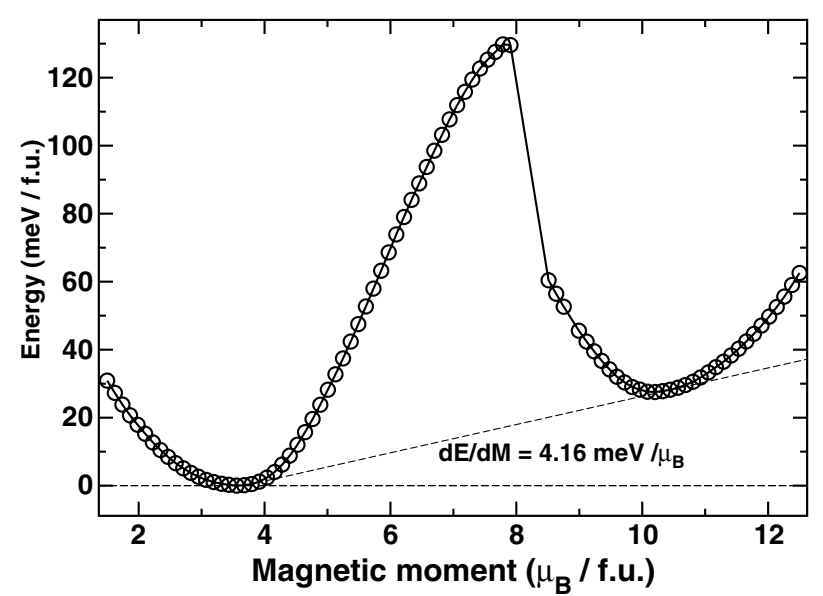

Figure 9. Total energy of $\mathrm{GdCo}_{9} \mathrm{Si}_{4}$ as a function of the total spin moment. The calculated data points are given by circles. The critical field $B_{\mathrm{c}}$ can be estimated from the slope $\mathrm{d} E / \mathrm{d} M$ of a common tangent construction shown by the dashed line.

$\left(M_{\text {calc }}=3.53 \mu_{\mathrm{B}}\right.$ versus $\left.M_{\text {exp }}=5.0 \mu_{M}\right)$ and (ii) for the exchange field $B_{\mathrm{c}}\left(B_{\mathrm{c}}^{\text {calc }}=71.7 \mathrm{~T}\right.$ versus $\left.B_{\mathrm{c}}^{\exp } \simeq 36 \mathrm{~T}\right)$. At present, it is unclear whether this cancellation in the framework of the FSM is of systematic nature or just by chance. To answer this question, further systematic investigations are necessary. Further small corrections for the calculated field $B_{1}$ can be expected due to the neglect of orbital magnetism in the FSM approach. Because the orbital moment of $\mathrm{Co}$ is expected to be larger for the ferromagnetic state, a slight reduction of the calculated $B_{1}$ is expected from this contribution [26].

\section{Conclusion}

Alloys prepared along the composition line $\mathrm{GdCo}_{13-x} \mathrm{Si}_{x}$ crystallize in bet $\mathrm{BaCd}_{11^{-}}$-type structure (1:11 phase) in the range of $x \sim 2.5$. A structural transition to bet $\mathrm{LaFe}_{9} \mathrm{Si}_{4}$ type structure (1:13 phase) occurs when $x \geqslant 3.8$. A rather narrow homogeneity region, $3.9 \leqslant x \leqslant 4.2$, is obtained for the tetragonal $1: 13$ phase, where $\mathrm{GdCo}_{9} \mathrm{Si}_{4}$ is a true ternary compound with a fully ordered distribution of $\mathrm{Gd}, \mathrm{Co}$ and $\mathrm{Si}$ atoms within the space group $\mathrm{I} / \mathrm{mcm}$.

The magnetic characterization of $\mathrm{GdCo}_{9} \mathrm{Si}_{4}$ by means of ac susceptibility, magnetization and specific heat measurements reveals a phase transition towards a ferrimagnetic state at $T_{\mathrm{C}} \simeq 47 \mathrm{~K}$, where the local moment $\mathrm{Gd}$ subsystem couples antiparallel to the itinerant ferromagnetism on the Co $3 \mathrm{~d}$ subsystem. The ferrimagnetic alignment of the $\mathrm{Co}$ and $\mathrm{Gd}$ subsystems breaks up above about $27 \mathrm{~T}$, in excellent agreement with the molecular field calculations based on a Néel model with the strongest coupling within the sublattice of $16 \mathrm{k}$ Co sites, $\gamma=90 \mathrm{G} \mathrm{mol} \mathrm{emu}^{-1}$, ferrimagnetic inter-sublattice coupling, $\gamma_{\mathrm{GdCo}}=$ $-9.8 \mathrm{G} \mathrm{mol} \mathrm{emu}^{-1}$, and negligible exchange coupling within the $\mathrm{Gd}$ subsystem.

The magnitude of the specific heat anomaly, $\Delta C \sim 8.5 \mathrm{~J} \mathrm{~mol}^{-1} \mathrm{~K}^{-1}$ is significantly smaller than that expected for a mean-field-like phase transition (e.g. $\Delta C \sim 20.15 \mathrm{~J} \mathrm{~mol}^{-1} \mathrm{~K}^{-1}$ for the $J=7 / 2$ ff moments of Gd) and thus indicative of the itinerant nature of the transition. This is also supported by the comparison of electrical resistivity data, $\rho(T)$, of $\mathrm{GdCo}_{9} \mathrm{Si}_{4}$ and $\mathrm{YCo}_{9} \mathrm{Si}_{4}$ with the very same qualitative behaviour with $\rho_{\text {mag }}(T) \propto T^{1.7}$ well below $T_{\mathrm{C}}$ and 
with a negative curvature of $\rho(T)$ in the paramagnetic state, which is an indication of strong spin fluctuations in both compounds.

By means of LSDA electronic structure calculations, we described the magnetic properties of $\mathrm{GdCo}_{9} \mathrm{Si}_{4}$ on a microscopic basis. Despite the strong spin fluctuations present in $\mathrm{GdCo}_{9} \mathrm{Si}_{4}$, we find a very good agreement of the calculated and the measured field $B_{1}$ for the onset of the non-collinear magnetic state.

\section{Acknowledgments}

This work was supported by the Austrian Science Foundation Funds under project P-16250Phy. HR was supported by the DFG (Emmy Noether program). MEl-H thanks TU Wien for the financial support during his stay. Funding of the high field experiments by the European Commission under contract RII3-CT-2004-506239 (EuroMagNET) is acknowledged.

\section{References}

[1] Rao G H, Liang J K, Zhang Y L, Cheng X R and Tang W H 1994 Appl. Phys. Lett. 641650

[2] Hu F X, Shen B G, Sun J R, Cheng Z H and Zhang X X 2000 J. Phys.: Condens. Matter 12 L691

[3] Fujieda F, Fujita A, Fukamichi K, Yamazaki Y and Lijima Y 2001 Appl. Phys. Lett. 79653

[4] Fujita A, Akamatsu Y and Fukamichi K 1999 J. Appl. Phys. 854756

[5] Michor H, El-Hagary M, Della Mea M, Pieper M W, Reissner M, Hilscher G, Khmelevskyi S, Mohn P, Schneider G, Giester G and Rogl P 2004 Phys. Rev. B $69081404(\mathrm{R})$

[6] Michor H, El-Hagary M, Özcan S, Horyn A, Bauer E, Reissner M, Hilscher G, Khmelevskyi S, Mohn P and Rogl P 2005 Physica B 359-361 1177

[7] El-Hagary M, Michor H, Wind M, Bauer E, Hilscher G and Rogl P 2004 J. Alloys Compounds 367239

[8] El-Hagary M, Michor M, Bauer E, Grössinger R, Kerschl P, Eckert D, Müller K-H, Rogl P, Giester G and Hilscher G 2005 Physica B 359-361 311

[9] Huang M Q, Wallace W E, Obermyer R T, Simizu S, McHenry M and Sankar S G 1996 J. Appl. Phys. 795949

[10] El-Hagary M, Michor H, Bauer E, Della Mea M, Hense K and Hilscher G 2004 J. Magn. Magn. Mater. 272-276 e 445

[11] El-Hagary M 2005 Egypt. J. Solids 2885

[12] Skolozdra R V, Shcherba I D, Bodak O I, Melnyk G A, Gorelenko Yu K, Yarovetz Y, Dobryanska L O and Lobojko V S 2000 J. Alloys Compounds 296272

[13] Krug H, Doerr M, Eckert D, Eschrig H, Fischer F, Fulde P, Grössinger R, Handstein A, Herlach F, Hinz D, Kratz R, Loewenhaupt M, Müller K-H, Pobell F, Schultz L, Siegel H, Steglich F and Verges P 2001 Physica B 294/295 605

[14] Heiba Z K, El-Hagary M, Michor H and Hilscher G 2006 Intermetallics 14220

[15] Tang W, Liang J, Chen X and Rao G 1994 J. Appl. Phys. 764095

[16] Pourarian F, Malik S K, Boltich E B, Sankar S G and Wallace W E 1989 IEEE Trans. Magn. 253315

[17] Rao G H, Liang J K, Zhang Y L, Cheng X R, Tang W H and Dong C 1995 Phys. Rev. B 5060

[18] Néel L 1948 Ann. Phys. 3137

[19] Smart J S 1966 Effective Field Theories of Magnetism (Philadelphia, PA: Saunders)

[20] Clark A E and Callen E 1968 J. Appl. Phys. 395972

[21] Steiner M J, Beckers F, Niklowitz P G and Lonzarich G G 2003 Physica B 329-333 1079

[22] Koepernik K and Eschrig H 1999 Phys. Rev. B 591743 web-reference: www.fplo.de

[23] Perdew J P and Wang Y 1992 Phys. Rev. B 4513244

[24] Eschrig H 1989 Optimized LCAO Method and the Electronic Structure of Extended Systems (Berlin: Springer)

[25] Leithe-Jasper A, Schnelle W, Rosner H, Senthilkumaran N, Rabis A, Baenitz M, Gippius A, Morozova E, Mydosh J A and Grin Y 2003 Phys. Rev. Lett. 91037208

[26] Kuz'min M D, Skourski Y, Eckert D, Rochter M, Müller K-H, Skokov K P and Tereshina I S 2004 Phys. Rev. B $\mathbf{7 0} 172412$ 